\title{
Electropolymerization of poly(3,4-ethylenedioxythiophene) onto polyvinyl alcohol- graphene quantum dot-cobalt oxide nanofiber composite for high-performance supercapacitor
}

\begin{abstract}
Fabrication of highly conductive nanofiber by coating polyvinyl alcohol-graphene quantum dot-cobalt oxide (PVA-GQD- $\mathrm{Co}_{3} \mathrm{O}_{4}$ ) nanofiber composite with a conductive material, poly(3,4-ethylenedioxythiophene) (PEDOT) for supercapacitor was successfully prepared via two-step technique i.e. electrospinning and electropolymerization. The prepared electrode materials were characterized using FTIR, Raman and XRD analysis to confirm the structure of the electrospun nanofiber composite. The presence of cauliflower-like structure studied by FESEM revealed that PEDOT was uniformly coated on PVA-GQD- $\mathrm{Co}_{3} \mathrm{O}_{4}$ electrospun nanofibers. The PVA-GQD- $\mathrm{Co}_{3} \mathrm{O}_{4} / \mathrm{PEDOT}$ nanofiber composite exhibited a specific capacitance of $361.97 \mathrm{~F} / \mathrm{g}$, low equivalent series resistance (ESR) and excellent stability with retention of $96 \%$ after 1000 cycles. The PVA-GQD-Co $\mathrm{O}_{3} / \mathrm{PEDOT}$ nanofiber composite also demonstrated a high specific energy and excellent specific power ranged from 16.51 to 19.98 $\mathrm{Wh} / \mathrm{kg}$ and $496.10-2396.99 \mathrm{~W} / \mathrm{kg}$, as the current density increased from 1.0 to $5.0 \mathrm{~A} / \mathrm{g}$.
\end{abstract}

Keyword: Supercapacitor; Graphene quantum dots; Cobalt oxide; Nanofiber; Electropolymerization 